\title{
Čerenkov-type second-harmonic generation in thin planar calix[4]arene waveguiding films
}

\author{
O. F. J. Noordman, N. F. van Hulst, and B. Bölger
}

Applied Optics Group, Faculty of Applied Physics and Micro-Electronics, Material Engineering, Sensors and Actuators Research Institute, University of Twente, P.O. Box 217, 7500 AE Enschede, The Netherlands

Received January 3, 1995; revised manuscript received July 10, 1995

\begin{abstract}
A new type of organic molecule, calix[4]arene, is applied in a second-harmonic-generating waveguide device for the first time. Linear optical properties of the calix[4]arene waveguiding film have been measured with the prism-coupling method. The molecules in the film are oriented by a corona-poling technique. With a Makerfringe experiment, the induced $d_{33}$ and $d_{31}$ at $\lambda=1064 \mathrm{~nm}$ are determined at 8.6 and $2.0 \mathrm{pm} / \mathrm{V}$, respectively. The calix[4]arene thin film has been applied to a Cerenkov-type device that generates second-harmonic light radiating into the glass substrate. The highest efficiency obtained is $0.23 \%$ for a fundamental wavelength of $820 \mathrm{~nm}$ by the use of fundamental power densities of $100 \mathrm{MW} / \mathrm{cm}^{2}$ in the waveguide and a device length of $6 \mathrm{~mm}$. A coupled-mode theory has been developed that can properly explain the experimentally observed second-harmonic-generating efficiency and the angle of radiation into the substrate. Two methods for the enhancement of device efficiency are proposed, both based on theoretical calculations with the coupled-mode theory. An enhancement of 2 orders of magnitude is feasible. (C) 1995 Optical Society of America
\end{abstract}

\section{INTRODUCTION}

In the early days of nonlinear optics, second-harmonic(SH-) generating experiments were performed in bulk crystals. The advantage of confining the light to a small area over long distances was realized by going from bulk crystals to inorganic waveguides, for example $\mathrm{LiNbO}_{3}$. However, the flexibility in fabricating such devices was poor, and therefore interest in organic materials as alternatives to inorganic crystals increased. Several polymer systems have been investigated for these purposes. ${ }^{1-6}$

In this paper the first SH-generating device based on a novel type of molecule, calix[4]arene, ${ }^{7}$ is presented. The conventional scheme of phase matching in both crystals and waveguides is critical with respect to several parameters, such as wavelength, temperature, refractive index, and layer thickness in the case of waveguides. ${ }^{8}$ An alternative that is much less critical to these parameters is to generate the $\mathrm{SH}$ in the Cerenkov scheme. ${ }^{9-11}$ The advantage of this scheme is that possible destructive interference is avoided because the $\mathrm{SH}$ is generated as a nonguided wave, which is automatically phase matched. However, compared with real phase matching of fundamental and $\mathrm{SH}$ guided modes, the efficiency of the Čerenkov process and the quality of the SH output beam are worse. As a first step to show the potential of calix[4]arenes in a device, it was chosen to fabricate a Čerenkov-type device. ${ }^{12}$

\section{THEORY}

The optical response of a material is generally described by the equation

$$
\mathbf{P}=\chi^{(1)} \cdot \mathbf{E}+\chi^{(2)}: \mathbf{E}^{2}+\chi^{(3)}: \mathbf{E}^{3}+, \ldots,
$$

where $\mathbf{E}$ is the electric field of the light beam that generates a polarization $\mathbf{P}$ in a material because of its first-, second-, third-, and higher-order susceptibilities $\chi^{(n)}$. In the case of SH generation, a polarization at frequency $2 \omega$ is induced by an electric field with frequency $\omega$, as in

$$
\mathbf{P}(2 \omega)=1 / 2 \chi^{(2)}(-2 \omega ; \omega, \omega): \mathbf{E}(\omega) \mathbf{E}(\omega)=\epsilon_{0} d \cdot \mathbf{E}(\omega)^{2}
$$

The third-rank tensor $\chi^{(2)}(-2 \omega ; \omega, \omega)$ can be written as a $3 \times 6$ matrix $d$ with 18 components. The SH process occurs only in noncentrosymmetric materials; consequently the organic nonlinear optical (NLO) molecules in a thin film must be oriented. This is done by the application of a static electric field, which induces the alignment of molecules with an electric dipole moment. The symmetry of such a system is $C_{\infty}$, which results in only five nonzero components of the $d$ matrix: $d_{33}, d_{31}, d_{32}, d_{15}$, and $d_{24}$. The last four components are equal when Kleinmann symmetry is assumed. Several models have been developed to describe the effect of a static electric field on the orientational distribution of the molecules. ${ }^{3,13}$ In the isotropic model, the distribution is given by a Boltzmann distribution of the energy of a dipole in a static electric field. If $\theta$ is the angle between the dipole axis and the applied static electric field, which is in the direction perpendicular to the film, the resulting $d_{33}$ and $d_{31}$ are

$$
\begin{aligned}
d_{33} & =1 /{ }_{2} N \beta_{z z z} f_{2 \omega} f_{\omega}^{2}\left\langle\cos ^{3}(\theta)\right\rangle, \\
d_{31} & =1 /{ }_{2} N \beta_{z z z} f_{2 \omega} f_{\omega}^{2}\left\langle 1 / 2 \cos (\theta) \sin ^{2}(\theta)\right\rangle \\
& =1 /{ }_{4} N \beta_{z z z} f_{2 \omega} f_{\omega}^{2}\left[\langle\cos (\theta)\rangle-\left\langle\cos ^{3}(\theta)\right\rangle\right], \\
f_{\omega} & =\frac{n^{2}(\omega)+2}{3},
\end{aligned}
$$

where $N$ is the number density, $\beta_{z z z}$ is the molecular hyperpolarizability, $f_{\omega}$ and $f_{2 \omega}$ are the Lorentz-Lorenz local field factors, $n(\omega)$ is the refractive index of the thin 




Fig. 1. Čerenkov SH-generating scheme with coordinate axes.

film, and $\langle\ldots\rangle$ indicates the averages of the orientational distribution of the molecules. The ratio $d_{33} / d_{31}$ following from Eqs. (3) is

$$
\frac{d_{33}}{d_{31}}=\frac{2\left\langle\cos ^{3}(\theta)\right\rangle}{\langle\cos (\theta)\rangle-\left\langle\cos ^{3}(\theta)\right\rangle},
$$

which can be calculated for a known distribution. For moderately oriented films, this ratio is close to 3 . It increases for increased alignment.

The Čerenkov device we consider is a planar waveguide that consists of a glass substrate and a single thin film of a NLO active organic material, as shown in Fig. 1. Light with the fundamental frequency $\omega$ is coupled into the waveguide as a guided mode by prism coupling. ${ }^{14,15}$ The polarization can be transversal electric (TE) or transversal magnetic (TM). Because of the second-order susceptibility of the film, a polarization with a frequency of $2 \omega$ is induced. If the phase velocity of this induced polarization is above the speed of light with frequency $2 \omega$ in the glass substrate, the $\mathrm{SH}$ light will radiate into the substrate. This criterion is met when $n_{\text {eff }}^{\omega}<n_{\text {substrate. The }}^{2 \omega}$ angle of radiation of the $\mathrm{SH}$ light with respect to the film, the Čerenkov angle $\theta_{\text {cer }}$, is given by

$$
\cos \theta_{\text {cer }}=\frac{n_{\text {eff }}^{\omega}}{n_{\text {substrate }}^{2 \omega}},
$$

which follows from the conservation of wave vectors in the $x$ direction. This automatic matching of wave vectors is the important advantage of generating the $\mathrm{SH}$ with the Čerenkov scheme.

To be able to predict device efficiencies, we have developed a coupled-mode theory for Čerenkov $\mathrm{SH}$ generation. ${ }^{16,17}$ The basic equation follows from Maxwell's equations:

$$
\begin{aligned}
\nabla \times\left(\nabla \times \mathbf{E}_{\nu}\right) & =-\mu_{0} \frac{\partial^{2}}{\partial t^{2}}\left(\bar{\epsilon}: \mathbf{E}_{\nu}+\mathbf{P}_{\mathrm{NLO}, \nu}\right), \\
\nu & =\omega, 2 \omega,
\end{aligned}
$$

where $\mathbf{E}_{\nu}$ is the electric field of the light with frequency $\nu$, $\bar{\epsilon}$ is the linear optical susceptibility of the material, and $\mathbf{P}_{\mathrm{NLO}}$ is the polarization at frequency $\nu$ generated from light with a different frequency. For small conversion efficiencies, pump depletion can be neglected, so only the equation for $2 \omega$, the $\mathrm{SH}$, needs to be evaluated.

The electric-field amplitudes of the light with frequencies $\omega$ and $2 \omega$ are described with a guided mode and a substrate-radiating mode, respectively. A general way of describing the electric and the magnetic fields in the $y$ direction for the TE- and the TM-polarized modes, respectively, is

$$
\begin{aligned}
\mathbf{E}_{\nu}(\mathbf{r}, t)= & \operatorname{Re}\left|A_{\nu}(x) E_{y}^{\nu}(z) \exp \left[i\left(\nu t-\beta_{\nu} x\right)\right] \mathbf{e}_{y}\right| \\
& (\mathrm{TE} \text { mode }), \\
\mathbf{H}_{\nu}(\mathbf{r}, t)=\operatorname{Re}\left|A_{\nu}(x) H_{y}^{\nu}(z) \exp \left[i\left(\nu t-\beta_{\nu} x\right)\right] \mathbf{e}_{y}\right| & (\mathrm{TM} \text { mode }), \\
\nu=\omega, 2 \omega . &
\end{aligned}
$$

The electric- and the magnetic-field vectors for the TE and the TM modes, respectively, are described with a normalized field distribution $E_{y}^{\nu}(z)$ and $H_{y}^{\nu}(z)$, the amplitude of the field $A_{\nu}$, and a factor that determines the propagation in the $x$ direction of the light of frequency $\nu$ with wave vector $\beta_{\nu}$. For guided modes, the energy in the $x$ direction, the direction of propagation, is conserved. The normalization of the field distribution of the fundamental guided mode is such that the power flow is $1 \mathrm{~W}$ in a $1-\mathrm{m}$-broad waveguide $(1 \mathrm{~W} / \mathrm{m})$.

The generated $\mathrm{SH}$ radiates into the substrate. The energy flow in the $x$ direction is therefore not conserved. This requires an extra normalization in the $x$ direction. The normalization is therefore chosen to be $1 \mathrm{~W} / 1 \mathrm{~m}^{2}$ of the waveguide.

For both types of mode the normalized fields in the $x$ and the $z$ directions are simple functions of the normalized fields in Eqs. (7):

$$
\begin{aligned}
& H_{x}^{\omega}(z)=\frac{-i}{\mu_{0} \omega} \frac{\partial E_{y}^{\omega}(z)}{\partial z} \\
& H_{z}^{\omega}(z)=\frac{\beta_{\omega}}{\mu_{0} \omega} E_{y}^{\omega}(z) \quad(\text { TE mode }) \\
& E_{x}^{\omega}(z)=\frac{i}{\omega \epsilon_{x}} \frac{\partial H_{y}^{\omega}(z)}{\partial z} \\
& E_{z}^{\omega}(z)=\frac{-\beta_{\omega}}{\omega \epsilon_{z}} H_{y}^{\omega}(z) \quad(\text { TM mode })
\end{aligned}
$$

In the case of a $\mathrm{SH}$ guided mode, changes in the $\mathrm{SH}$ intensity would be due to the coupling with the fundamental beam. However, in the SH-radiating mode there is a large intrinsic loss of energy into the substrate because of the radiative character. The total change in the $\mathrm{SH}$ intensity is given by

$$
\begin{aligned}
\frac{\partial A_{2 \omega}(x)}{\partial x} & =\left|\frac{\partial A_{2 \omega}(x)}{\partial x}\right|_{\mathrm{NLO}}+\left|\frac{\partial A_{2 \omega}(x)}{\partial x}\right|_{\mathrm{rad}}, \\
\left|\frac{\partial A_{2 \omega}(x)}{\partial x}\right|_{\mathrm{rad}} & =-\frac{A_{2 \omega}(x)}{l_{d}},
\end{aligned}
$$

where changes that are due to the coupling from the fundamental beam (NLO) and changes that are due to the radiative loss into the substrate (rad) are distinguished. The substrate loss is linear in the mode amplitude $A_{2 \omega}(x)$ and can be expressed in an effective decay length $l_{d}$, which follows from the normalized field distribution and conservation of energy:

$$
\begin{aligned}
& \int_{-\infty}^{h} \int_{0}^{2 \pi / 2 \omega}(\mathbf{E} \times \mathbf{H})_{x} \mathrm{~d} t \mathrm{~d} z=\frac{l_{d}}{2} \\
& \Rightarrow \int_{-\infty}^{h}\left(E_{y}^{2 \omega}\right)^{2} \mathrm{~d} z=l_{d} \frac{\mu_{0} 2 \omega}{\beta_{2 \omega}} \quad \text { (TE mode) }, \\
& \int_{-\infty}^{h} \frac{\left(H_{y}^{2 \omega}\right)^{2}}{\epsilon_{z}} \mathrm{~d} z=l_{d} \frac{2 \omega}{\beta_{2 \omega}} \quad \text { (TM mode) }
\end{aligned}
$$


The double integral evaluates the pointing vector of the $\mathrm{SH}$-radiating mode in the $x$ direction in the region in or close to the waveguiding layers.

At the start of the waveguide, $A_{2 \omega}$ is 0 and starts increasing because of the coupling from the fundamental beam. However, after the SH light has propagated typically 20 to $100 \mu \mathrm{m}$, depending on $l_{d}$, an equilibrium $A_{2 \omega, \text { equi }}$ is reached, where $\partial A_{2 \omega}(x) / \partial x=0$, which results in

$$
\left|\frac{\partial A_{2 \omega}(x)}{\partial x}\right|_{\mathrm{NLO}}=\frac{A_{2 \omega, \text { equi }}}{l_{d}}
$$

As a result, the overall $\mathrm{SH}$ intensity can be written as

$$
P_{2 \omega}=\int_{-\infty}^{\infty} \int_{-\infty}^{\infty}\left[A_{2 \omega}(y, x)\right]^{2} \mathrm{~d} y \mathrm{~d} x \approx\left(A_{2 \omega, \text { equi }}\right)^{2} b L
$$

where $b$ is the beam width and $L$ is the device length.

We calculate the equilibrium value of $A_{2 \omega}$ by substituting Eqs. (7) into Eqs. (6) and by using Eq. (11). This results in

$$
\begin{aligned}
\frac{A_{2 \omega, \text { equi }}}{l_{d}} E_{y}^{2 \omega}(z)= & \frac{-i 2 \mu_{0} \omega^{2}}{\beta_{2 \omega}} P_{\text {ind }, y}(2 \omega) \quad \text { (TE mode), } \\
\frac{A_{2 \omega, \text { equi }}}{l_{d}} H_{y}^{2 \omega}(z)= & i \omega P_{\text {ind }, z}(2 \omega) \\
& +\frac{\omega n_{z, 2 \omega}^{2}}{\beta_{2 \omega}} \frac{\partial}{\partial z}\left[\frac{1}{n_{x, 2 \omega}^{2}} P_{\text {ind }, x}(2 \omega)\right]
\end{aligned}
$$

(TM mode),
The value of $A_{2 \omega}$, equi for $\mathrm{TE}_{\omega} \Rightarrow \mathrm{TM}_{2 \omega}$ conversion becomes

$$
\begin{aligned}
A_{2 \omega, \text { equi }}= & A_{\omega}^{2}\left\{i \beta_{\omega} \int_{-\infty}^{\infty} \frac{H_{y}^{2 \omega}(z)}{n_{z, 2 \omega}^{2}} d_{32}(z)\left[E_{y}^{\omega}(z)\right]^{2} \mathrm{~d} z\right. \\
& +i \mu_{0} \omega \int_{-\infty}^{\infty} \frac{H_{y}^{2 \omega}(z)}{n_{x, 2 \omega}^{2}} d_{12}(z) E_{y}^{\omega}(z) H_{x}^{\omega}(z) \mathrm{d} z \\
& \left.+\frac{1}{2} \sum_{i=1}^{n-1} H_{y}^{2 \omega}\left(z_{i}\right) \Delta_{i, i+1}\left(d_{12} \frac{1}{n_{x, 2 \omega}^{2}}\right)\left[E_{y}^{\omega}\left(z_{i}\right)\right]^{2}\right\}
\end{aligned}
$$

The last term is a summation over the interfaces between the different layers involved in the waveguide. This is caused by the term

$$
\frac{\omega n_{z, 2 \omega}^{2}}{\beta_{2 \omega}} \frac{\partial}{\partial z}\left[\frac{1}{n_{x, 2 \omega}^{2}} P_{\text {ind }, x}(2 \omega)\right]
$$

in Eqs. (13). The induced polarization is not continuous at an interface between two materials with different values of the involved $d$ component. This results in a delta function at the interfaces when the differentiation with respect to $z$ is carried out. However, when the equation is integrated it becomes a step function that can be summed over the present interfaces.

For $\mathrm{TM}_{\omega} \Rightarrow \mathrm{TM}_{2 \omega}$ conversion, the value of $A_{2 \omega \text {, equi }}$ becomes

$$
\begin{aligned}
A_{2 \omega, \text { equi }}= & A_{\omega}^{2}\left[i \beta_{\omega} \int_{-\infty}^{\infty} \frac{H_{y}^{2 \omega}(z)}{n_{z, 2 \omega}^{2}}\left\{d_{31}(z)\left[E_{x}^{\omega}(z)\right]^{2}+d_{33}(z)\left[E_{z}^{\omega}(z)\right]^{2}+2 d_{35}(z) E_{x}^{\omega}(z) E_{z}^{\omega}(z)\right\} \mathrm{d} z\right. \\
& +\int_{-\infty}^{\infty} \frac{H_{y}^{2 \omega}(z)}{n_{x, 2 \omega}^{2}}\left(d_{11}(z) E_{x}^{\omega}(z) \frac{-i}{\omega \epsilon_{0} n_{x, \omega}^{2}} k_{\omega}^{2}(z) H_{y}^{\omega}(z)+d_{13}(z) E_{z}^{\omega}(z) i \beta_{\omega} \frac{n_{x, \omega}^{2}}{n_{z, \omega}^{2}} E_{x}^{\omega}(z)\right. \\
& \left.+d_{15}(z)\left\{i \beta_{\omega} \frac{n_{x, \omega}^{2}}{n_{z, \omega}^{2}}\left[E_{x}^{\omega}(z)\right]^{2}+\frac{-i}{\omega \epsilon_{0} n_{x, \omega}^{2}} k_{\omega}^{2}(z) H_{y}^{\omega}(z) E_{z}^{\omega}(z)\right\}\right) \mathrm{d} z \\
& \left.+\frac{1}{2} \sum_{i=1}^{n-1} H_{y}^{2 \omega}\left(z_{i}\right) \Delta_{i, i+1}\left(\frac{1}{n_{x, 2 \omega}^{2}}\left\{d_{11}\left[E_{x}^{\omega}\left(z_{i}\right)\right]^{2}+d_{13}\left[E_{z}^{\omega}\left(z_{i}\right)\right]^{2}+2 d_{15} E_{x}^{\omega}\left(z_{i}\right) E_{z}^{\omega}\left(z_{i}\right)\right\}\right)\right] .
\end{aligned}
$$

where $\beta_{2 \omega}$ is the wave vector in the $x$ direction, which equals $2 \beta_{\omega}, E_{y}^{2 \omega}(z)$ and $H_{y}^{2 \omega}(z)$ are the normalized fields, $n$ is the refractive index of the material, and $P_{\text {ind }, x}(2 \omega), P_{\text {ind, } y}(2 \omega)$, and $P_{\text {ind, } z}(2 \omega)$ are the amplitudes of the polarizations in the $x$, the $y$, and the $z$ directions, respectively, which are induced by the fundamental guided mode. These polarizations can be expressed in the normalized distributions of the fundamental guided mode [Eqs. (7) and (8)], its mode amplitude $A_{\omega}$, and a number of $d$ components. Next, both sides of Eqs. (13) are multiplied by either $E_{y}^{2 \omega}$ or $H_{y}^{2 \omega}$ in the cases of TE or TM, respectively, and the equations are integrated over the $z$ coordinate, which causes canceling of the decay length $l_{d}$ when Eqs. (10) are used.

For a thin film with $C_{\infty_{\nu}}$ symmetry, the $d$ components necessary for conversion to TE-polarized SH modes are all 0 . Therefore we consider the conversion to TM-polarized $\mathrm{SH}$ modes.
Here $k_{\omega}$ is a wave vector in the $z$ direction that is used to describe the field distribution of the fundamental guided mode.

For a given interaction length $L$, width of the fundamental beam $b$, and power $P_{\omega}$, the $\mathrm{SH}$ power $P_{2 \omega}$ becomes

$$
P_{2 \omega}=P_{\omega}^{2} \frac{L}{b}\left(\ldots \int_{-\infty}^{\infty} \cdots \sum_{i=1}^{n-1} \ldots\right)^{2}
$$

The efficiency of the SH process is clearly dependent on not only the fundamental beam power, beam width, and device length, but also on the value of the square of the overlap integral, which is determined by the values of the $d$ components and the distribution of the normalized electric and magnetic fields. How this can influence the efficiency is shown in Fig. 2. The product of the SH-radiation mode, the $d_{33}$, and the square of the fundamental guided mode has both a positive part and 


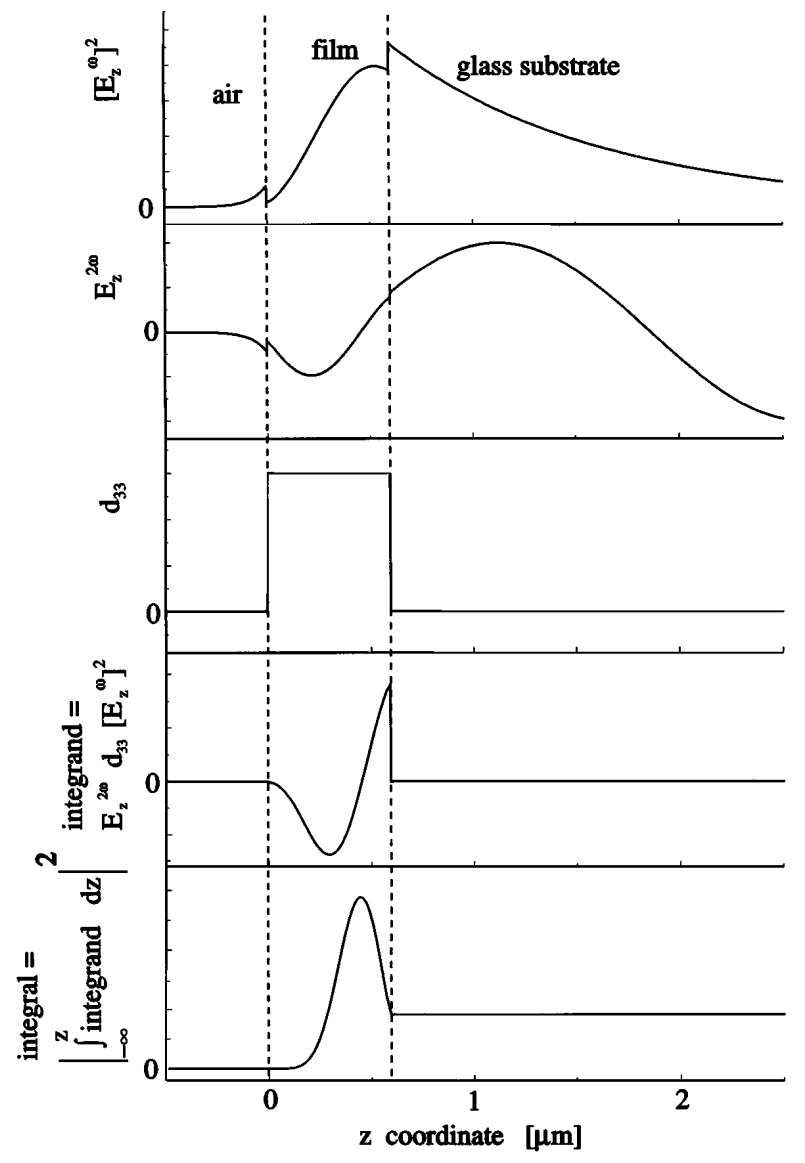

Fig. 2. Field distributions in a Čerenkov device and their influence on the overlap integral.

a negative part, which cause the condition that the overlap integral is not optimized and even can become 0 for certain thicknesses. This is a well-known phenomena in coupled-mode theory for phase matching between guided modes and clearly also holds for the Cerenkov coupled mode as described in this paper.

\section{MATERIALS}

The NLO active material used is one of a new class of molecules called calix[4]arenes. ${ }^{7}$ The structure of tetranitro-tetrapropoxycalix[4]arene, the molecular derivative under investigation, is shown in Fig. 3. It has a large molecular hyperpolarizability ${ }^{18} \beta_{z z z}(\lambda=1064 \mathrm{~nm})$ of $28 \pm 3 \times 10^{-30} \mathrm{esu}$ that is due to the presence of four $D-\pi-A$ systems in one molecule, which also causes a large static dipole moment $\mu_{0}$ of $13.8 \mathrm{D}$. However, the absorption maximum is still in the UV at $291 \mathrm{~nm}$, and the transparency cutoff is at approximately $400 \mathrm{~nm}$. This makes it a suitable material for frequency-doubling laser diodes in the 800-900-nm wavelength region.

The tetranitro-tetrapropoxycalix[4]arene, which from now on is called simply calix[4]arene, can be made into a thin film with the spincoating technique, when it is dissolved in chloroform. ${ }^{19,20}$ A thickness sufficient for waveguiding can be realized by the addition of $25 \mathrm{wt} . \%$ polymer [poly(phenyl methacrylate) (PPMA)]. The alignment of the molecules in a static electric field is done by means of the corona-poling technique ${ }^{21}$ at elevated temperatures $\left(100^{\circ} \mathrm{C}\right)$ under atmospheric conditions for 15 min, as shown in Fig. 4.

The second-order susceptibility of the thin films has been measured with a Maker-fringe setup ${ }^{22}$ that compares the angular-dependent $\mathrm{SH}$ signal with the signal from a quartz reference $\left(d_{11}=0.51 \mathrm{pm} / \mathrm{V}\right.$ at $\lambda=$ $1064 \mathrm{~nm}$ ). We measure $d_{31}$ and $d_{33}$ separately by first using an $s$-polarized fundamental beam and detecting the $p$-polarized $\mathrm{SH}$ that is generated only by the $d_{31}$ of the film and second by using a $p$-polarized fundamental beam and detecting again the $p$-polarized $\mathrm{SH}$ light, in which $d_{32}\left(=d_{31}\right), d_{24}\left(=d_{31}\right)$, and $d_{33}$ contribute to the $\mathrm{SH}$ signal. ${ }^{23}$ With $d_{31}$ known from the $s \rightarrow p$ experiment, the remaining contribution of $d_{33}$ to the $p \rightarrow p$ experiment can be calculated. Characteristic values measured for 75 wt. \% calix[4]arene/PPMA films are given in Table 1. A resonance enhancement of the $d$ values with a factor of

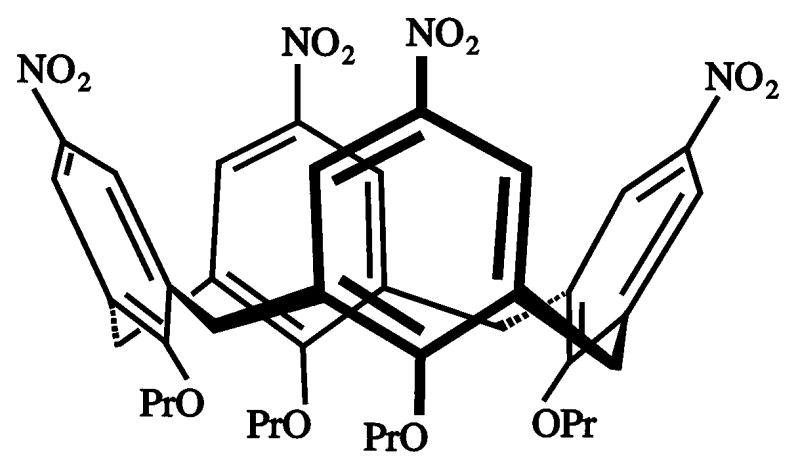

Fig. 3. Structure of the tetranitro-tetrapropoxycalix[4]arene.

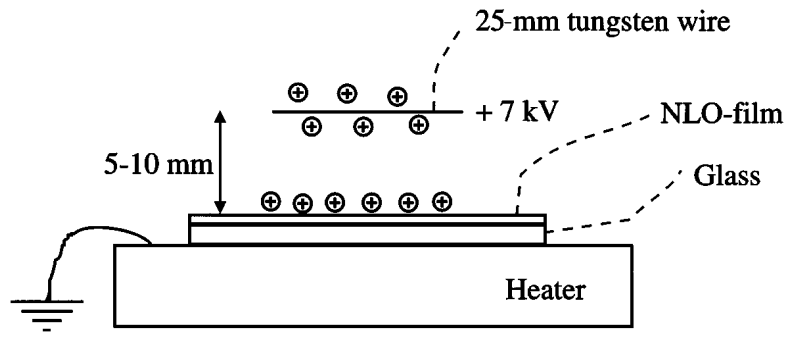

Fig. 4. Corona-poling setup.

Table 1. $d_{33}, d_{31}, d_{33} / d_{31}$ Ratio, and $\left\langle\cos ^{3} \theta\right\rangle$ Alignment Parameter Calculated from $d_{33} / d_{31}$ with the Isotropic Model and Calculated from the Molecular $\beta_{z z z}$ and $d_{33}$ for Two Different Corona-Poled 75 wt. \% Calix[4]arene/PPMA Thin Films

\begin{tabular}{|c|c|c|c|c|c|c|}
\hline Film (Number) & $\lambda(\mathrm{nm})$ & $d_{33}(\mathrm{pm} / \mathrm{V})$ & $d_{31}(\mathrm{pm} / \mathrm{V})$ & $d_{33} / d_{31}$ & $\begin{array}{c}\left\langle\cos ^{3} \theta\right\rangle \\
\text { from } d_{33} / d_{31}\end{array}$ & $\begin{array}{c}\left\langle\cos ^{3} \theta\right\rangle \text { from } \\
d_{33}, N, \beta_{z z z}, f_{\omega}, f_{2 \omega}\end{array}$ \\
\hline 1 & 1064 & $6.8 \pm 0.3$ & $1.9 \pm 0.1$ & $3.6 \pm 0.2$ & $0.36 \pm 0.05$ & $0.48 \pm 0.10$ \\
\hline 2 & 1064 & $8.6 \pm 0.4$ & $2.0 \pm 0.1$ & $4.3 \pm 0.3$ & $0.47 \pm 0.04$ & $0.60 \pm 0.10$ \\
\hline 2 & 820 & $12.2 \pm 0.6$ & $3.2 \pm 0.2$ & $3.8 \pm 0.3$ & & \\
\hline
\end{tabular}




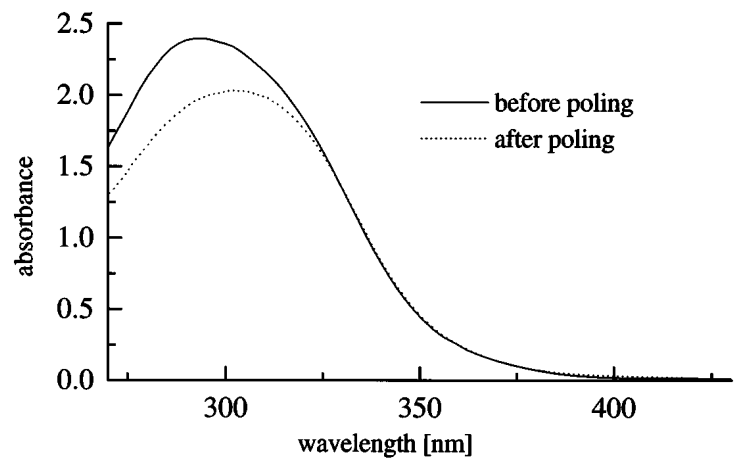

Fig. 5. Absorption spectra of 75 wt. \% calix[4]arene/PPMA thin film before and after poling at $7 \mathrm{kV}$ at $100^{\circ} \mathrm{C}$ for $15 \mathrm{~min}$.

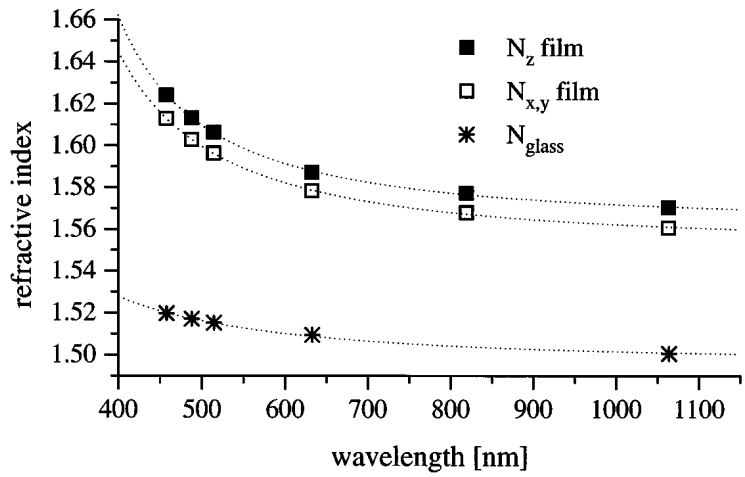

Fig. 6. Refractive indices of the corona-poled $75 \mathrm{wt} . \%$ calix[4]arene/PPMA thin film perpendicular and parallel to the film and the glass substrate.

1.5 for a wavelength closer to the absorption of the film is observed.

The ratio $d_{33} / d_{31}$ turns out to be larger than 3 . The difference in ratio for film 2 for the two wavelengths can be explained by the fact that the $\mathrm{SH}$ wavelength for a fundamental wavelength of $820 \mathrm{~nm}$ is so close to the absorption band that Kleinmann symmetry is no longer valid. The values of the alignment parameter $\left\langle\cos ^{3} \theta\right\rangle$ calculated from the $d_{33} / d_{31}$ ratio with the isotropic model do not correspond exactly with the values calculated with Eq. (3a) by the use of the $d_{33}$ value at $\lambda=1064 \mathrm{~nm}$, the molecular $\beta_{z z z}$, the number density $N$, and the estimated Lorentz-Lorenz local field factors $f_{\omega}$ and $f_{2 \omega}$. This indicates that the distribution of the molecules is different from the distribution in the isotropic model. This is not unexpected, as the percentage of NLO molecules is much more than $10 \%$ and the system cannot be considered to be a guest-host system.

After some initial decay in the first week after poling, the $d$ values remain stable at $65 \%$ of their initial values at room temperature and at $20 \%$ at $100{ }^{\circ} \mathrm{C} .{ }^{20}$

The effect of the dc electric-field poling on the orientation of the molecules also results in a decrease of the absorption of the film, ${ }^{24}$ as shown in Fig. 5. The absorption maximum shows a small shift to the red. The transparency cutoff, however, remains at approximately $400 \mathrm{~nm}$.

\section{EXPERIMENT}

To calculate theoretical efficiencies and to design an efficient device, the refractive indices of the materials incor- porated in the waveguide have to be known. These were measured by the prism-coupling method for a wide range of wavelengths, as shown in Fig. 6. The variation in refractive index between different calix[4]arene/PPMA thin films was less than 0.005 . For the corona-poled films, differences in the refractive indices parallel and perpendicular to the film up to 0.014 were measured. This birefringence is because of the alignment of the dipoles, which results in a higher refractive index in the $z$ direction.

The indices were fitted with the Sellmeier equation,

$$
n^{2}(\lambda)=A^{2}+B \frac{\lambda^{2}}{\lambda^{2}-\lambda_{0}^{2}}
$$

Typical values of the coefficients for 75 wt. $\%$ calix[4]arene/PPMA and the glass substrate are given in Table 2.

The Čerenkov experiment was done with a $Q$-switched Nd:YAG laser, operating at $10 \mathrm{~Hz}$ and generating 8$10-n s$ pulses, as the source of fundamental light. In combination with a dye laser after frequency doubling the 1064-nm light, fundamental light of $820 \mathrm{~nm}$ was generated at $10 \mathrm{~Hz}$ with 6 -ns-width pulses.

The fundamental light was coupled into the waveguide by an SF6 prism. The fundamental beam power $P_{\omega}$ inside the waveguide was determined by a second prism that coupled out the light, which was subsequently detected with a photodiode. The output end of the glass was polished so that the Cerenkov angle of the SH light that radiated into the glass could be accurately measured. The intensity was measured with a photodiode.

The first experiment was done with a $78 \mathrm{wt} \%$ calix[4]arene/PPMA film with a thickness of $675 \pm 10 \mathrm{~nm}$ and a fundamental wavelength of $1064 \mathrm{~nm}$. The generated green $\mathrm{SH}$, given in Table 3 , could be seen by eye. An unusual fact emerged, in that the $\mathrm{SH}$ intensity was a factor of 10 higher for the TE-polarized fundamental beam than for the TM-polarized beam, while the main

Table 2. Sellmeier Coefficients for a Corona-Poled 75 wt.\% Calix[4]arene/PPMA Thin Film and Glass Substrate

\begin{tabular}{lccc}
\hline Parameter & Film $n_{z}$ & Film $n_{x, y}$ & $n_{\text {glass }}$ \\
\hline$A$ & 1.4649 & 1.4270 & 1 \\
$B$ & 0.2976 & 0.3761 & 1.2403 \\
$\lambda_{0}(\mathrm{~nm})$ & 288 & 265 & 106 \\
\hline
\end{tabular}

Table 3. Experimental Values, Calculated Efficiencies, and Overlap Integrals [From Eq. (16)] for the Cerenkov

Experiment on a $675 \pm 10 \mathrm{~nm}$ thick, $78 \mathrm{wt} \%$ Corona-Poled Calix[4]arene/PPMA Film ${ }^{a}$

\begin{tabular}{lcc}
\hline \multirow{2}{*}{\multicolumn{1}{c}{ Parameter }} & \multicolumn{2}{c}{ Mode } \\
\cline { 2 - 3 } \multicolumn{1}{c}{ TM } & TE \\
\hline Čerenkov angle $(\mathrm{deg})$ & $5.3 \pm 0.5$ & $3.9 \pm 0.5$ \\
$P_{\omega}(\mathrm{kW})$ in waveguide & $3.3 \pm 0.5$ & $3.2 \pm 0.5$ \\
$P_{2 \omega}(\mathrm{W})$ SH power & $0.028 \pm 0.003$ & $0.29 \pm 0.03$ \\
Efficiency $\left(\times 10^{4}\right)$ & $0.085 \pm 0.015$ & $0.91 \pm 0.17$ \\
Overlap $^{2}\left(\mathrm{~W}^{-1}\right)\left(\times 10^{8}\right)$ & $0.051 \pm 0.020$ & $0.59 \pm 0.24$ \\
\hline
\end{tabular}

${ }^{a} \lambda=1064 \mathrm{~nm}$, device length $L=5.0 \pm 0.5 \mathrm{~mm}$, and beam width $b=$ $1.0 \pm 0.2 \mathrm{~mm}$. 




Fig. 7. Experimental and theoretical values for the Čerenkov angle and the overlap integral for a $675 \pm 10 \mathrm{~nm}$ thick, $78 \mathrm{wt} . \%$ calix[4]arene/PPMA corona-poled film at $\lambda=1064 \mathrm{~nm}$.

contributing $d_{33}$ for TM was a factor of 3.5 larger than the $d_{32}\left(=d_{31}\right)$, which contributes to the TE case. This could be explained by the coupled-mode theory. From the fundamental beam power $P_{\omega}$, the SH power $P_{2 \omega}$, the beam width $b$, and the device length $L$, an experimental value for the square of the overlap integral in Eq. (16) was calculated. In Fig. 7 the measured Cerenkov angle and the overlap integral are shown together with the theoretical values that were calculated as a function of film thickness, based on the measured refractive index of the film, the index of the substrate, and the measured $d_{33}$ and $d_{31}$ values. The correspondence between theory and experiment turns out to be good. The square of the overlap integral for the TE-polarized fundamental beam is indeed approximately a factor of 10 higher for the specific thickness of this film.

To verify the theory and to increase the $\mathrm{TM}_{\omega} \Rightarrow \mathrm{TM}_{2 \omega}$ conversion efficiency, we performed experiments on two other samples with different thicknesses $(591 \pm 10$ and $599 \pm 10 \mathrm{~nm}$ ). The experimental data are given in Tables 4 and 5, and the comparisons with theory are shown in Figs. 8 and 9. Although the percentage of calix[4]arenes in both films was the same, the theoretical curves for the Čerenkov angle and the overlap integral were somewhat different because of different $d$ values and small differences in the refractive indices. For the experiment in Table 5 and Fig. 9, the two available fundamental wavelengths, 1064 and $820 \mathrm{~nm}$, were used.

The highest efficiency of $0.23 \%$ was measured for $\mathrm{TM}_{\omega} \Rightarrow \mathrm{TM}_{2 \omega}$ conversion with $\lambda=820 \mathrm{~nm}$, which is, in our opinion, a good result for Čerenkov SH from a planar waveguide. The correspondence between theory and experiment is good for the Čerenkov angle. The overlap integral shows reasonable correspondence when we introduce a correction factor for the fundamental intensity: losses in the waveguide cause the measured fundamental intensity, after outcoupling with the prism, to be lower than the average intensity in the waveguide. The correction factor takes these losses into account. The difference in the correction factors, 1.4 versus 1.7 , is caused mainly by the difference in quality of the film.

\section{DISCUSSION}

In Figs. 7-9 the theoretical curves show a minimum in $\mathrm{TM}_{\omega} \Rightarrow \mathrm{TM}_{2 \omega}$ conversion with $\lambda=1064 \mathrm{~nm}$ for a thickness of $\sim 670-710 \mathrm{~nm}$. This minimum is explained by the fact that the SH field distribution has both a negative part and a positive part, which cancel each other exactly in the minimum. The reason why the overlap integral is a factor of 10 larger for $\lambda=820 \mathrm{~nm}$ than for $\lambda=1064 \mathrm{~nm}$ in the case of $\mathrm{TM}_{\omega} \Rightarrow \mathrm{TM}_{2 \omega}$ conversion is also explained by the field distributions. For $\lambda=1064 \mathrm{~nm}$, a much larger part of the field is canceled than for $\lambda=820 \mathrm{~nm}$.

Table 4. Experimental Values, Calculated Efficiencies, and Overlap Integrals for the Čerenkov Experiment on a $591 \pm 10 \mathrm{~nm}$ thick, 75 wt. \% Corona-Poled Calix[4]arene/PPMA Film ${ }^{a}$

\begin{tabular}{lcc}
\hline \multirow{2}{*}{\multicolumn{1}{c}{ Parameter }} & \multicolumn{2}{c}{ Mode } \\
\cline { 2 - 3 } \multicolumn{1}{c}{ TM } & TE \\
\hline Čerenkov angle (deg) & $7.3 \pm 0.5$ & $6.8 \pm 0.5$ \\
$P_{\omega}(\mathrm{kW})$ in waveguide & $2.1 \pm 0.2$ & $1.44 \pm 0.14$ \\
$P_{2 \omega}(\mathrm{W})$ SH power & $0.069 \pm 0.006$ & $0.028 \pm 0.003$ \\
Efficiency $\left(\times 10^{4}\right)$ & $0.33 \pm 0.05$ & $0.19 \pm 0.03$ \\
Overlap $^{2}\left(\mathrm{~W}^{-1}\right)\left(\times 10^{8}\right)$ & $0.31 \pm 0.10$ & $0.27 \pm 0.09$ \\
\hline
\end{tabular}

${ }^{a} \lambda=1064 \mathrm{~nm}$, device length $L=5.0 \pm 0.5 \mathrm{~mm}$, and beam width $b=$ $1.0 \pm 0.2 \mathrm{~mm}$.

Table 5. Experimental Values, Calculated Efficiencies, and Overlap Integrals for the Čerenkov Experiment on a $599 \pm 10 \mathrm{~nm}$ thick, 75 wt.\% Corona-Poled Calix[4]arene/PPMA Film ${ }^{a}$

\begin{tabular}{|c|c|c|c|c|}
\hline \multirow{3}{*}{$\begin{array}{l}\text { Parameter } \\
\text { Mode }\end{array}$} & \multicolumn{4}{|c|}{ Wavelength (nm) } \\
\hline & \multicolumn{2}{|c|}{1064} & \multicolumn{2}{|c|}{820} \\
\hline & TM & $\mathrm{TE}$ & TM & $\mathrm{TE}$ \\
\hline Čerenkov angle (deg) & $7.5 \pm 0.5$ & $6.1 \pm 0.5$ & $5.1 \pm 0.5$ & $4.7 \pm 0.5$ \\
\hline$P_{\omega}(\mathrm{kW})$ in waveguide & $1.21 \pm 0.12$ & $3.4 \pm 0.3$ & $1.0 \pm 0.1$ & $0.78 \pm 0.08$ \\
\hline$P_{2 \omega}(\mathrm{W}) \mathrm{SH}$ power & $0.30 \pm 0.03$ & $0.12 \pm 0.01$ & $2.3 \pm 0.2$ & $0.24 \pm 0.02$ \\
\hline Efficiency $\left(\times 10^{4}\right)$ & $2.5 \pm 0.4$ & $0.35 \pm 0.05$ & $23 \pm 3$ & $3.1 \pm 0.4$ \\
\hline $\operatorname{Overlap}^{2}\left(\mathrm{~W}^{-1}\right)\left(\times 10^{8}\right)$ & $3.4 \pm 1.1$ & $0.17 \pm 0.05$ & $38 \pm 12$ & $6.6 \pm 2.0$ \\
\hline
\end{tabular}

${ }^{a}$ Device length $L=6.0 \pm 0.5 \mathrm{~mm}$, and beam width $b=1.0 \pm 0.2 \mathrm{~mm}$. 


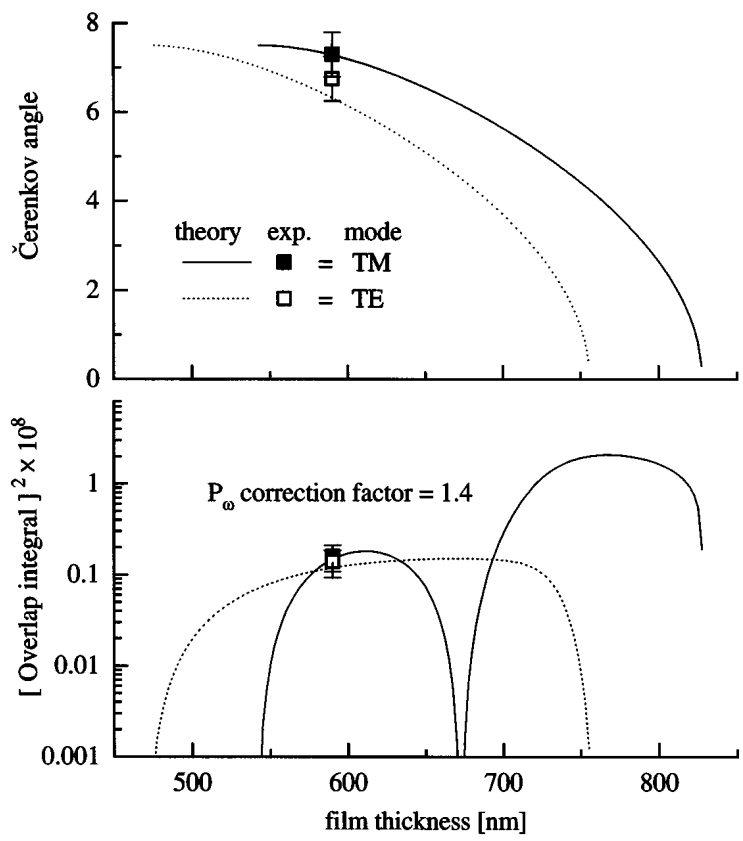

Fig. 8. Experimental and theoretical values for the Čerenkov angle and the overlap integral for a $591 \pm 10 \mathrm{~nm}$ thick, 75 wt. \% calix[4]arene/PPMA corona-poled film at $\lambda=1064 \mathrm{~nm}$.

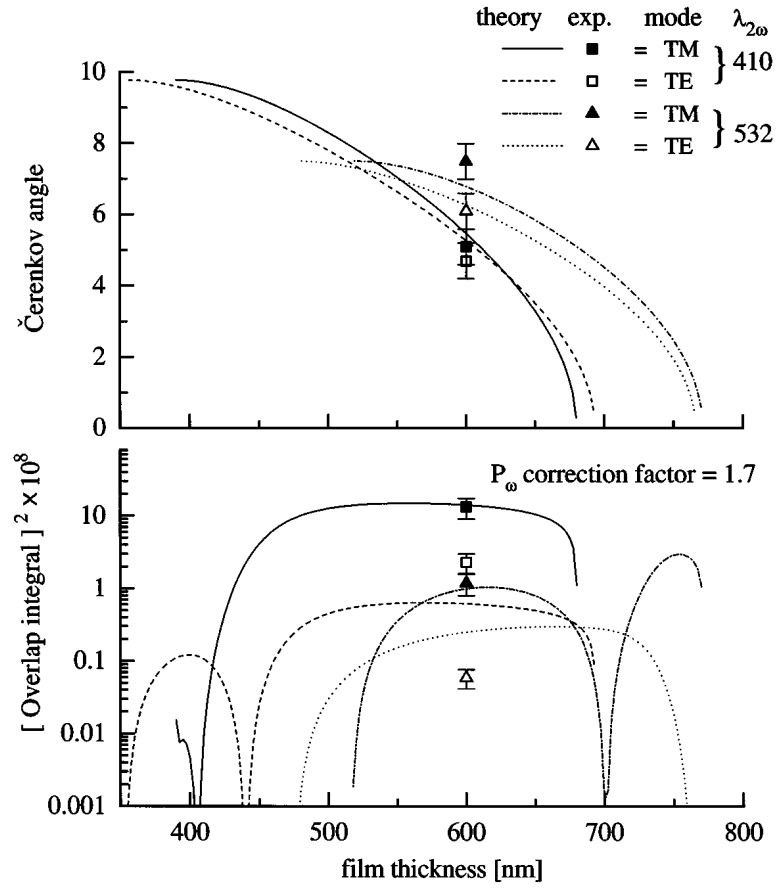

Fig. 9. Experimental and theoretical values for the Cerenkov angle and the overlap integral for a $599 \pm 10 \mathrm{~nm}$ thick, $75 \mathrm{wt}$. \% calix[4]arene/PPMA corona-poled film at $\lambda=1064$ and $820 \mathrm{~nm}$.

The overlap integral and therefore also the efficiency could be significantly increased if either the positive part or the negative part of the integrand would be 0 . A way to do this would be to bleach the $d$ values for part of the calix/PPMA film. It would be even better if the $d$ value of part of the film could be reversed in sign such that the integrand has the same sign for the entire film. The field distributions and the resulting overlap integral for the three different situations are shown in Fig. 10 for $\lambda=1064 \mathrm{~nm}$. For simplicity in the calculations, only the $d_{33}$ value is taken to be nonzero. The $d_{33}$ has by far the largest contribution to the overlap integral in the case of $\mathrm{TM}_{\omega} \Rightarrow \mathrm{TM}_{2 \omega}$ conversion. In Fig. 11, theoretical curves for these three situations are shown. The maximum overlap integral shows an increase with approximately a factor of 30 in the case of bleaching the $d$ value and even 2 orders of magnitude for partially reversing the sign of the $d$ value. The same calculations were done for a fundamental wavelength of $820 \mathrm{~nm}$. The enhancement in the maximum overlap integral in that case was a factor 6 .

In the experiment it is, however, difficult to realize partial bleaching or sign reversal of the $d$ values. It would be better to introduce an extra passive waveguiding layer that can have a similar effect as bleaching the $d_{33}$.

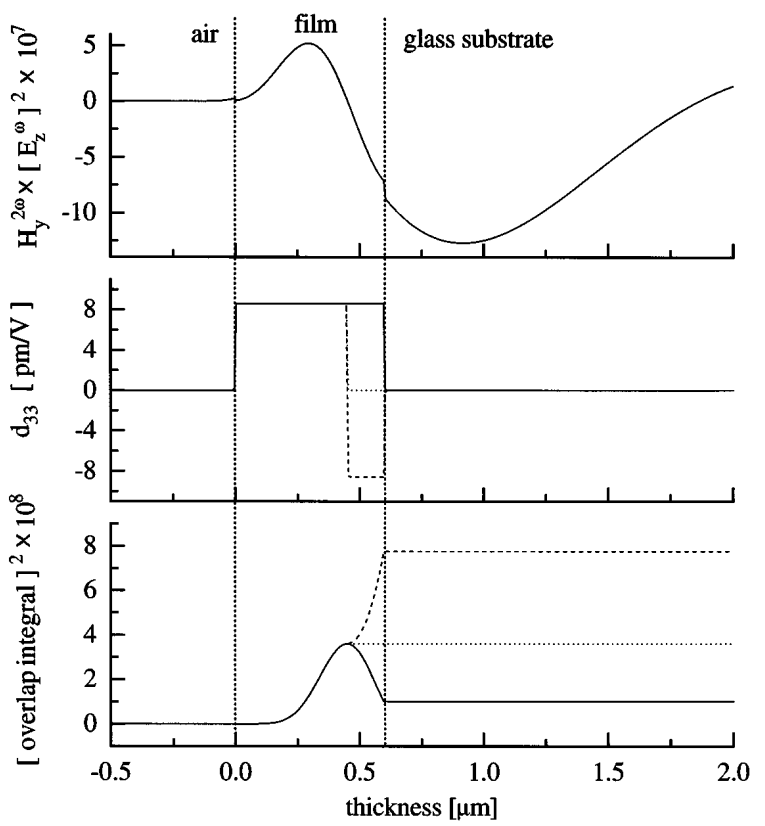

Fig. 10. Influence of the distribution and the sign of the $d_{33}$ value on the overlap integral.

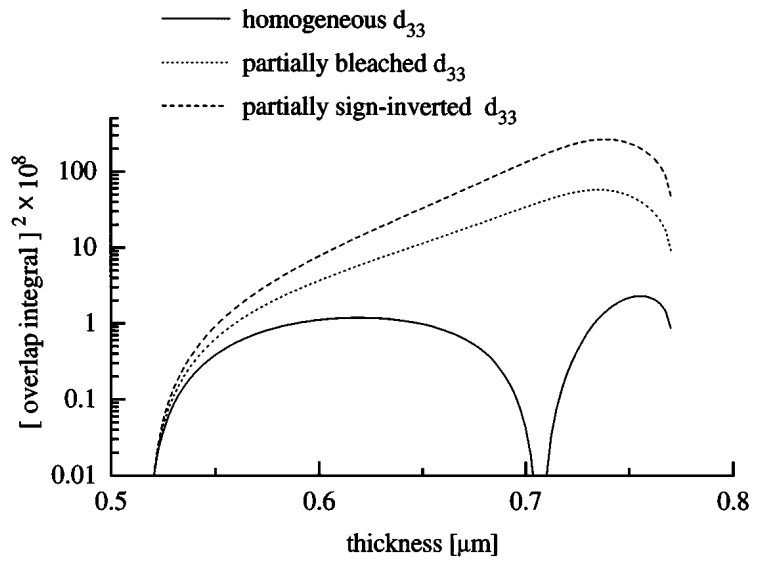

Fig. 11. Enhancement of the overlap integral by the bleaching of or the inversion of the sign of the $d_{33}$ value. 


\section{CONCLUSIONS}

A number of experiments were done on three different waveguiding films of $78 \mathrm{wt} \%$ and $75 \mathrm{wt} \%$ calix[4]arene/PPMA on a glass substrate. The experimentally observed Čerenkov angle and the SH intensity show good agreement with the developed coupled-mode theory. Small differences in the SH intensity can be ascribed to the losses in the film. The maximum achieved efficiency was $0.23 \%$. From theory it is shown that the efficiency can be significantly enhanced just by modification of the $d_{33}$ value inside the NLO film.

In the experiments described the beam width was $1 \mathrm{~mm}$. However, if we narrow this width down to $5 \mu \mathrm{m}$ by introducing a channel, the maximum normalized efficiency, when a partially sign-inverted film is used, is estimated with the theory for planar waveguides to be of the order of $0.16 \% \mathrm{~W}^{-1} \mathrm{~cm}^{-1}$. This is nearly 3 orders of magnitude better than the highest experimentally observed value of $3.8 \times 10^{-4} \% \mathrm{~W}^{-1} \mathrm{~cm}^{-1}$. However, when a laser diode $(0.1 \mathrm{~W})$ is used, the efficiency will be much below $0.1 \%$, which is still low for practical applications. Therefore future work will be aimed at realizing a device with phase matching between fundamental and $\mathrm{SH}$ guided modes, which can result in much higher efficiencies according to the coupled-mode theory, but that is much more critical in its behavior toward the device parameters.

\section{ACKNOWLEDGMENTS}

The authors thank E. Kelderman and D. N. Reinhoudt for making available the calix[4]arene. This research was supported by the Micro Optics program of the Onderzoeks Stimulerings Fonds of the University of Twente.

\section{REFERENCES}

1. D. J. Williams, "Organische polymere und nichtpolymere Materialien mit guten nichtlinearen optische eigenschaften," Angew. Chem. 96, 637-651 (1984).

2. J. Zyss, "Nonlinear organic materials for integrated optics: a review," J. Mol. Electron. 1, 25-45 (1985).

3. D. S. Chemla and J. Zyss, eds., Nonlinear Optical Properties of Organic Molecules and Crystals (Academic, Orlando, Fla., 1987), Vol. 1, Chap. II-7.

4. G. L. J. A. Rikken, C. J. E. Seppen, S. Nijhuis, and E. W. Meijer, "Poled polymers for frequency doubling of diode lasers," Appl. Phys. Lett. 58, 435-437 (1991).

5. D. M. Burland, R. D. Miller, O. Reiser, R. J. Twieg, and C. A. Walsh, "The design, synthesis, and evaluation of chromophores for second harmonic generation in a polymer waveguide," J. Appl. Phys. 71, 410-417 (1992).

6. G. L. J. A. Rikken, C. J. E. Seppen, A. H. J. Venhuizen, S. Nijhuis, and E. G. J. Staring, "Frequency doubling of diode lasers with poled polymers," Philips J. Res. 46, 215-230 (1992).

7. E. Kelderman, L. Derhaeg, G. J. T. Heesink, W. Verboom, J. F. Engbersen, N. F. van Hulst, A. Persoons, and D. N.
Reinhoudt, "NitroCalix[4]arenes as molecules for secondorder nonlinear optics," Angew. Chem. Int. Ed. Engl. 31, 1075-1077 (1992).

8. B. Chen, C. L. Tang, and J. M. Telle, "CW harmonic generation in the uv using a thin-film waveguide on a nonlinear substrate," Appl. Phys. Lett. 25, 495-498 (1974).

9. P. K. Tien, R. Ulrich, and R. J. Martin, "Optical second harmonic generation in form of coherent Cerenkov radiation from a thin-film waveguide," Appl. Phys. Lett. 17, 447-450 (1970).

10. O. Sugihara, S. Kunioka, Y. Nonaka, R. Aizawa, Y. Koike, T. Kinoshita, and K. Sasaki, "Second harmonic generation by Cerenkov-type phase matching in a poled polymer waveguide,” J. Appl. Phys. 70, 7249-7252 (1991).

11. K. Clays, N. J. Armstrong, and T. L. Penner, "Blue and green Cerenkov-type second-harmonic generation in a polymeric Langmuir-Blodgett waveguide," J. Opt. Soc. Am. B 10, $886-893$ (1993).

12. O. F. J. Noordman, N. F. van Hulst, and B. Bölger, "Cerenkov second-harmonic radiation from organic calix[4]arenes thin films," in Conference on Lasers and Electro-Optics Europe (Optical Society of America, Washington, D.C., 1994), paper CWL6, pp. 274-275.

13. C. P. J. M. van der Vorst and S. J. Picken, "Electric field poling of acceptor-donor molecules," J. Opt. Soc. Am. B 7, 320-325 (1990).

14. P. K. Tien, R. Ulrich, and R. J. Martin, "Modes of propagating light waves in thin deposited semiconductor films," Appl. Phys. Lett. 14, 291-294 (1969).

15. R. Ulrich, "Theory of the prism-film coupler by plane-wave analysis," J. Opt. Soc. Am. 60, 1337-1350 (1970).

16. H. Tamada, "Coupled-mode analysis of second harmonic generation in the form of Cerenkov radiation from a planar optical waveguide," IEEE J. Quantum Electron. 27, 502-508 (1991).

17. N. Hashizume, T. Kondo, T. Onda, N. Ogasawara, S. Umegaki, and R. Ito, "Theoretical analysis of Cerenkov-type optical second-harmonic generation in slab waveguides," IEEE J. Quantum Electron. 28, 1798-1815 (1992).

18. G. J. T. Heesink, A. G. T. Ruiter, N. F. van Hulst, and B. Bölger, "Determination of hyperpolarizability tensor components by depolarized hyper Rayleigh scattering," Phys. Rev. Lett. 71, 999-1002 (1993).

19. G. J. T. Heesink, N. F. van Hulst, B. Bölger, E. Kelderman, J. F. Engbersen, W. Verboom, and D. N. Reinhoudt, "Novel calixarenes in thin films for efficient second harmonic generation," Appl. Phys. Lett. 62, 2015-2017 (1993).

20. E. Kelderman, G. J. T. Heesink, L. Derhaeg, T. Verbiest, P. T. A. Klaasse, W. Verboom, J. F. Engbersen, N. F. van Hulst, K. Clays, A. Persoons, and D. N. Reinhoudt, "Highly ordered films of neat calix[4]arenes for second order nonlinear optics," Adv. Mater. 5, 925-930 (1993).

21. J. R. Li and H. J. Wintle, "Unipolar corona space charge in wire-plane geometry: a first principles numerical computation," J. Appl. Phys. 65, 4617-4624 (1989).

22. J. Jerphagnon and S. K. Kurtz, "Maker fringes: a detailed comparison of theory and experiment for isotropic and uniaxial crystals," J. Appl. Phys. 41, 1667-1681 (1970).

23. L. M. Hayden, G. F. Sauter, F. R. Ore, P. L. Pasillas, J. M. Hoover, G. A. Lindsay, and R. A. Henry, "Second-order nonlinear optical measurements in guest-host and side-chain polymers," J. Appl. Phys. 68, 456-465 (1990).

24. R. H. Page, M. C. Jurich, B. Beck, A. Sen, R. J. Twieg, J. D. Swalen, G. C. Bjorklund, and C. G. Wilson, "Electrochromic and optical waveguide studies of corona-poled electro-optic polymer films,” J. Opt. Soc. Am. B 7, 1239-1250 (1990). 